\title{
SMOKING STATUS AFFECTING SURVIVAL OF ADENOCARCINOMA LUNG CANCER PATIENTS IN KUALA LUMPUR, MALAYSIA
}

\author{
NIDA SAJID ALI BANGASH ${ }^{1}$, NATASHA HASHIM ${ }^{2}$, NAHLAH ELKUDSSIAH ISMAIL ${ }^{3 *}$
}

${ }^{1}$ Clinical BioPharmaceutics Research Group, Faculty of Pharmacy, Universiti Teknologi MARA, Puncak Alam Campus, 42300 Bandar Puncak Alam, Selangor, Malaysia. ²Department of Oncology and Radiotherapy, Hospital Kuala Lumpur, 50300 Kuala Lumpur, Malaysia. ${ }^{3}$ Dean's office, Faculty of Pharmacy, MAHSA University, Jalan SP2, Bandar Saujana Putra, 42610 Jenjarom Kuala Langat, Selangor, Malaysia. Email: elkudssiah77@yahoo.com

Received: 17 January 2017, Revised and Accepted: 02 June 2017

\section{ABSTRACT}

Objective: Adenocarcinoma (AC) of the lung is now the most common histologic type of non-small cell lung cancer (NSCLC) worldwide since the past 20 years. This study was conducted to investigate survival difference among smoker and non-smoker lung AC patients.

Methods: A retrospective observational study was conducted for 81 advanced NSCLC adult Malaysian patients in Radiotherapy and Oncology Clinic at Hospital Kuala Lumpur, Malaysia. A total of adult 30 Malaysian smokers and 51 non-smokers with lung AC were included. Ex-smokers were not included in the study. Demographic and clinical data were collected and described. For survival analysis, Kaplan-Meier test and log-rank test were used to calculate overall survival (OS) and analyse the difference in the survival curve. Cox proportional hazard model was used to identify prognostic significance of smoking status.

Results: Non-smokers showed a significant association with female gender and Stage IV NSCLC. The median OS was higher for non-smokers (493 days) as compared to smokers (230 days). The Cox proportional hazard model showed higher hazard ratio for smokers.

Conclusion: Non-smoking is an independent positive prognostic factor in lung AC.

Keywords: Adenocarcinoma, Smokers, Survival.

(C) 2017 The Authors. Published by Innovare Academic Sciences Pvt Ltd. This is an open access article under the CC BY license (http://creativecommons. org/licenses/by/4. 0/) DOI: http://dx.doi.org/10.22159/ajpcr.2017.v10i9.17147

\section{INTRODUCTION}

Lung cancer is becoming one of the leading causes of cancer-related mortality worldwide [1]. The vast majority of lung cancer cases are attributed to smoking [2]. The smoking epidemic has been steadily decreased in developed countries whereas augmenting in underdeveloped and non-developed countries [3]. Non-small cell lung cancer (NSCLC) accounted for almost $80 \%$ of all lung cancer cases, comprising several histological types, including adenocarcinoma (AC), squamous cell carcinoma (SCC), and large cell carcinoma (LCC) [4]. Over the past 20 years, AC lung cancer had dramatically replaced SCC as the most frequent histologic type of $20 \%$ of lung cancer cases to approximately $40 \%$ [5]. The possible factor among smokers being the most frequent histologic type NSCLC included changes in the smoking trend from high-tar to low-tar filtered cigarettes [6]. Low-tar filtered cigarette result in deep and intense inhalation of tobacco smoke leading to greater delivery of carcinogens such as nitrogen oxide and nitro salted compounds to the lung periphery [7]. Passive smoking contributed to the shift in the cell type of NSCLC among non-smokers as a result of rapid inhalation of gaseous components of cigarette smoke into the deeper parts of the lungs as compared to the mainstream smoke containing more particulates [8].

This study was conducted to glance for the survival differences among smokers and non-smokers in lung AC. Since the mechanism of carcinogenesis of AC arising in smokers and non-smokers is distinct, it would imply differences in demographic and clinical characteristics and hence survival.

\section{METHODS}

This was a retrospective review of patients with NSCLC diagnosed at Radiotherapy and Oncology Clinic, Hospital Kuala Lumpur, Malaysia, from January 2009 to December 2012. The study was approved for research ethics by Medical Research and Ethics Committee, Ministry of Health, Malaysia. Patients' $\geq 18$ years old histopathologically confirmed to have locally advanced or metastatic AC were included in the analysis. Retrospectively collected demographic and clinical variables included gender, age (at diagnosis), stage of NSCLC, and smoking status. Data regarding smoking status included current smokers and non-smokers whereas ex-smokers were not included in the study. For survival analysis, each patient's time began on the date of diagnosis of disease and ended on the date of last follow-up for living patients and date of death for dead patients. Survival time was calculated in days. Furthermore, cancer-specific mortality was documented. Deaths resulting from any non-cancer causes were excluded from the study.

Statistical analysis was performed using SPSS 21.0 version. Demographic and clinical data were analysed descriptively by dividing the study population into two cohorts; smokers and non-smokers. Pearson $\chi^{2}$ tests were used to compare demographic and clinical variables among groups based on smoking status. The primary end point of this study was overall survival (OS). Survival was calculated using Kaplan-Meier test and compared using log-rank test. Prognostic importance of smoking status in lung $\mathrm{AC}$ was analysed using Cox proportional hazard model. A two-sided $\mathrm{p}<0.001, \mathrm{p}<0.05$ were considered statistically significant.

\section{RESULTS}

Characteristics of study Malaysian population categorised as smokers and non-smokers are listed in Table 1. There were 30 smokers and 51 non-smokers out of total 81 NSCLC AC patients $337.0 \%$ and $63.0 \%$, respectively). There were more male smokers $(32.0 \%)$ than male non-smokers (18.5\%). Most patients in both groups were between 
Table 1: Demographic and medical variables of patients

\begin{tabular}{lll}
\hline Characteristics & Smoker's n (\%) & Non-smoker's n (\%) \\
\hline $\begin{array}{l}\text { Total patients (n=81) } \\
\text { Gender }\end{array}$ & $30(37.0)$ & $51(63.0)$ \\
$\quad$ Male & $26(32.0)$ & $15(18.5)$ \\
$\quad$ Female & $4(5.0)$ & $36(44.4)$ \\
Age (years old) & & \\
$\quad$ 18-40 & $2(2.4)$ & $5(6.1)$ \\
$41-64$ & $24(29.6)$ & $38(47.0)$ \\
$\geq 65$ & $4(5.0)$ & $8(10.0)$ \\
NSCLC stage & & \\
III A & $4(5.0)$ & $2(2.4)$ \\
III B & $9(11.1)$ & $6(7.4)$ \\
IV & $17(21.0)$ & $43(53.0)$ \\
\hline
\end{tabular}

NSCLC: Non-small cell lung cancer

Table 2: Overall survival of smokers versus non-smokers

\begin{tabular}{llll}
\hline Smoking status & Median OS (days) & $\mathbf{9 5 \%}$ CI & p \\
\hline Smoker & 230 & $115.092-344.908$ & $0.039^{*}$ \\
Non-smoker & 493 & $262.284-723.716$ & \\
\hline
\end{tabular}

OS: Overall survival, CI: Confidence interval, ${ }^{*} \mathrm{p}<0.05$

Table 3: Survival analysis by Cox proportional hazard model

\begin{tabular}{lll}
\hline Parameter & HR (95\% CI) & p \\
\hline Male versus female gender & $0.914(0.509-1.643)$ & 0.764 \\
Smoker versus non-smoker & $1.865(1.022-3.406)$ & $0.042^{*}$ \\
Increasing age & $0.836(0.433-1.614)$ & 0.593 \\
\hline
\end{tabular}

HR: Hazard ratio, CI: Confidence interval, ${ }^{*} \mathrm{p}<0.05$

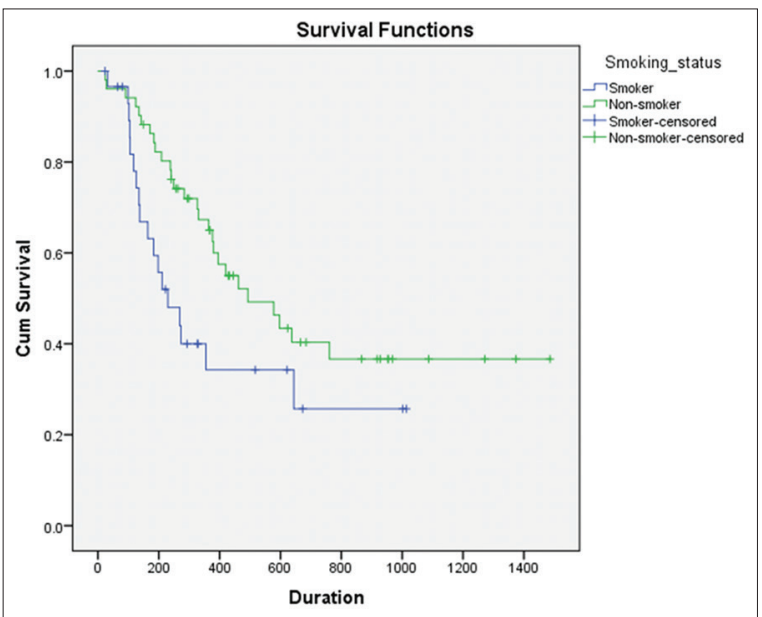

Fig. 1: Kaplan-Meier overall survival curve for smoker versus non-smoker

41 and 64 years old $(29.6 \%$ smokers vs. $47.0 \%$ non-smokers) and having Stage IV NSCLC (21.0\% smoker vs. $53.0 \%$ non-smokers). There were statistically significantly higher percentages of female $(p<0.001)$ and Stage IV NSCLC $(\mathrm{p}=0.022)$ patients in the non-smokers category. Median OS was 230 days for smokers and 493 days for non-smokers $(\mathrm{p}=0.039)$ (Table 2). Kaplan-Meier survival curve for smokers versus non-smokers is shown in Fig. 1. The hazard ratio (HR) for smokers was 1.865 times higher as compared to non-smokers $(p=0.042)$ (Table 3).

\section{DISCUSSION}

It is presumed that carcinogenic and mutagenic chemicals in the cigarette smoke induce lung carcinogenesis in smokers. However, non-smokers dictate distinct natural history and mechanism of carcinogenesis. The analysis was performed to determine disparities in patient characteristics and their survival among smokers and nonsmokers with lung AC.

The results of this study showed that females having lung AC were more likely to be non-smokers as compared to males. A similar result was also reported by a West Japan Oncology Group study that lung AC patients were mostly to be female non-smokers [9]. The statistically higher percentage of Stage IV NSCLC in non-smokers might be because this study comprised a higher proportion of Stage IV $(74.0 \%)$ and nonsmoker (63.0\%) AC patients. OS was longer, and HR to death was less for non-smokers as compared to smokers. Similarly, a prospective study reported that non-smoker lung AC patients had improved OS and nonsmoking showed positive prognostic significance in lung AC [10].

\section{CONCLUSION}

In conclusion, this study demonstrated that smoking status has a prognostic impact in patients with lung AC.

\section{REFERENCES}

1. Jemal A, Thomas A, Murray T, Thun M. Cancer statistics, 2002. CA Cancer J Clin 2002;52(1):23-47.

2. Carbone D. Smoking and cancer. Am J Med 1992;93 Suppl 1:13-7.

3. Parkin DM, Sasco AJ. Lung cancer: Worldwide variation in occurrence and proportion attributable to tobacco use. Lung Cancer 1993;9(1-6):1-16.

4. NSCLC: A Guide for Patients-Information based on ESMO Clinical Practice Guidelines. European Society for Medical Oncology (ESMO); 2014. Available from: http://www.esmo.org/EN-Non-Small-Cell-LungCancer-Guide-for-Patients. [Last cited on 2016 Jan 11]

5. Franceschi S, Bidoli E. The epidemiology of lung cancer. Ann Oncol 1999;10 Suppl 5:S3-6.

6. Kreuzer M, Kreienbrock L, Müller KM, Gerken M, Wichmann E. Histologic types of lung carcinoma and age at onset. Cancer 1999;85(9):1958-65.

7. Hoffmann D, Rivenson A, Hecht SS. The biological significance of tobacco-specific N-nitrosamines: Smoking and adenocarcinoma of the lung. Crit Rev Toxicol 1996;26(1):199-211.

8. Wynder EL, Goodman MT. Smoking and lung cancer: Some unresolved issues. Epidemiol Rev 1983;5(1):177-207.

9. Kogure Y, Ando M, Saka H, Chiba Y, Yamamoto N, Asami K, et al. Histology and smoking status predict survival of patients with advanced non-small-cell lung cancer. Results of West Japan Oncology Group (WJOG) Study 3906L. J Thorac Oncol 2013;8(6):753-8.

10. Nordquist LT, Simon GR, Cantor A, Alberts WM, Bepler G. Improved survival in never-smokers vs. Current smokers with primary adenocarcinoma of the lung. Chest 2004;126(2):347-51 